\title{
Retocolite ulcerativa e trombose venosa cerebral: relato de caso
}

\author{
Bárbara Fontes Macedo ${ }^{\text {; }}$ Lucas Renan Schaefer; Rafaela \\ Brambilla; Joana Carolina Junqueira Brum; Rômulo Gomes da \\ Silveira;
}

\section{Resumo}

A retocolite ulcerativa (RCU) é um tipo de doença intestinal inflamatória (DII), de etiologia desconhecida, que pode apresentar-se como uma doença confinada ao reto ou estender-se proximalmente, envolvendo todo colon. Os pacientes com DII tem risco aumentado de tromboembolismo, que incluem trombose venosa profunda, embolismo pulmonar, trombose venosa cerebral e raramente, trombose arterial. A trombose venosa cerebral é uma complicação rara porem potencialmente devastadora de DII. Este diagnóstico deve ser suspeito sempre em pacientes com sintomas neurológicos. Será relato o caso de um paciente do sexo masculino com alteração visual e cefaleia com diagnostico prévio de RCU. Após ressonância magnética do crânio, evidenciando trombose venosa no seio transverso e sigmóide, com extensão para veia jugular ipsilateral.

Palavras Chave: Trombose venosa cerebral; retocolite ulcerativa; alteração visual

1 Residente em medicina interna pela Ulbra; email: babsmacedo@hotmail.com 


\section{Introdução}

A retocolite ulcerativa (RCU) é um tipo de doença intestinal inflamatória(DII), de etiologia desconhecida, que pode apresentar-se como uma doença confinada ao reto ou estender-se proximalmente, envolvendo todo colon. Pode afetar qualquer grupo etário, porém os picos principalmente afetados são entre 15-30 e 50-70 anos.

Menos da metade dos pacientes apresentam sintomas extra-intestinais.

Os pacientes com DII tem risco aumentado de tromboembolismo, que incluem trombose venosa profunda, embolismo pulmonar, trombose venosa cerebral $\mathrm{e}$ raramente, trombose arterial.

A trombose venosa cerebral é uma complicação rara porem potencialmente devastadora de DII. Este diagnóstico deve ser suspeito sempre em pacientes com sintomas neurológicos.

A incidência da trombose venosa cerebral é de $6 \%$ em paciente com DII, sendo mais comum na RCU do que na doença de Chron.

Será relatado o caso de um paciente de 20 anos do sexo masculino, com diagnóstico prévio de retocolite ulcerativa em uso de mesalazina, que procurou atendimento devido a alterações visuais.

\subsection{Paciente:}

Paciente de 20 anos do sexo masculino, previamente diagnosticado com retocolite ulcerativa em tratamento irregular com mesalazina, procura atendimento devido a diplopia e cefaleia. Referia cefaleia em peso, contínua, com vômitos associado.

Evoluiu com parestesia em hemiface direita, irradiava para membro superior direito. Realizada ressonância magnética do crânio, onde havia alterações no seio transverso e sigmoide à direita com extensão para veia jugular ipsilateral, relacionado a trombose venosa em fase subaguda; defeito de enchimento dos seios transverso, sigmóide, veia jugular direita e também do seio sagital superior, relacionados a trombose.

\section{Método}

Após avaliação clínica, exame físico e ressonância magnética do crânio, de alterações visuais e cefaleia, diagnosticado trombose seio transverso, sagital superior e sigmóide a direita com extensão para veia jugular ipsilateral. 


\title{
3. Resultados
}

Após o diagnóstico de trombose evidenciando por exame de ressonância magnética, iniciado anticoagulação plena.

\section{Conclusões}

Em pacientes que sejam portadores de doenças inflamatórias intestinais com quadros de sinais e/ou sintomas neurológicos, deve-se ter como hipótese diagnóstica trombose, visto que é uma complicação rara porém potencialmente grave.

\section{Ulcerative Colitis and cerebral venous thrombosis: a case report}

\begin{abstract}
Ulcerative colitis (UC), a type of inflammatory bowel disease (IBD) of unknown etiology, which may present as a disease confined to the rectum or extend proximally, involving the entire colon. Patients with IBD are at increased risk of thromboembolism including deep vein thrombosis, pulmonary embolism, cerebral venous thrombosis and rarely, arterial thrombosis. Cerebral venous thrombosis is a rare complication however potentially devastating DII. This diagnosis should be suspected when patients with neurological symptoms. It will report the case of a male patient with visual and headache change with previous diagnosis of UC. After magnetic resonance image, revealed venous thrombosis in the transverse and sigmoid sinus, extending to the ipsilateral jugular vein.
\end{abstract}

Keywords: cerebral venous thrombosis; ulcerative colitis; visual change 


\section{Referências}

1. Talbot RW, Heppell J, Dozois RR, Beart RW., Jr Vascular complications of inflammatory bowel disease. Mayo Clin Proc. 1986;61:140-5. [PubMed]

2. Ozdil S, Akyuz F, Pinarbasi B, Demir K, Karaca C, Boztas G, et al. Ulcerative colitis: Analyses of 116 cases (do extraintestinal manifestations effect the time to catch remission?) Hepatogastroenterology. 2004;51:768-70. [PubMed]

3. Ameri A, Bousser MG. Cerebral venous thrombosis. Neurol Clin. 1992;10:87-111. [PubMed] 